\title{
AN AVERAGING PRINCIPLE FOR A COMPLETELY INTEGRABLE STOCHASTIC HAMILTONIAN SYSTEM
}

\author{
XUE-MEI LI
}

\begin{abstract}
We investigate the effective behaviour of a small transversal perturbation of order $\epsilon$ to a completely integrable stochastic Hamiltonian system, by which we mean a stochastic differential equation whose diffusion vector fields are formed from a completely integrable family of Hamiltonian functions $H_{i}, i=1, \ldots n$. An averaging principle is shown to hold and the action component of the solution converges, as $\epsilon \rightarrow 0$, to the solution of a deterministic system of differential equations when the time is rescaled at $1 / \epsilon$. An estimate for the rate of the convergence is given. In the case when the perturbation is a Hamiltonian vector field, the limiting deterministic system is constant in which case we show that the action component of the solution scaled at $1 / \epsilon^{2}$ converges to that of a limiting stochastic differentiable equation.
\end{abstract}

\section{INTRODUCTION}

The Model. A smooth $2 n$-dimensional manifold $M$ is said to be a symplectic manifold if it is equipped with a symplectic structure, that is, a closed differential two-form $\omega$ which is nondegenerate in the sense that for each $x \in M, \omega(v, w)=0$ for all $w \in T_{x} M$ implies $v=$ 0 . Equivalently $M$ admits a set of coordinates mapping such that the coordinate changing maps are symplectic on $\mathbf{R}^{2 n}$ with the standard symplectic form $\omega_{0}=\sum d p_{i} \wedge d q_{i}$.

A family of $n$ smooth Hamiltonians $\left\{H_{k}\right\}$ on a $2 n$ dimensional symplectic manifold is said to form a (completely) integrable system if they are pointwise Poisson commuting and if the corresponding Hamiltonian vector fields $X_{H_{k}}$ are linearly independent at almost all points. Given such an integrable family and a $C^{1}$ locally Hamiltonian vector field $V$ commuting with the family of vector fields $X_{H_{k}}$ in the sense of $\omega\left(X_{H_{k}}, V\right)=0$, consider the following model, which we call a completely integrable stochastic symplectic/Hamiltonian system:

$$
d x_{t}=\sum_{k=1}^{n} X_{H_{k}}\left(x_{t}\right) \circ d B_{t}^{k}+V\left(x_{t}\right) d t
$$

Here $\left(B_{t}^{k}, k=1, \ldots, n\right)$ are pairwise independent Brownian motions on a filtered probability space $\left(\Omega, \mathcal{F}, \mathcal{F}_{t}, P\right)$ with the standard assumptions on the filtration and $\circ$ stands for Stratonovitch integration. We have suppressed the chance element $\omega$ here as is conventional. Note that the customary symbol for the symplectic form is unfortunately the same as that for the chance variable, however confusion should not arise as the chance variable will from now on not be explicitly expressed unless indicated otherwise. We call respectively $X_{H_{k}}$ the diffusion vector fields and $V$ the drift vector field for the stochastic differential equation.

2000 Mathematics Subject Classification. Primary 60H10, 58J65; Secondary $58 \mathrm{~J} 37$.

Key words and phrases. Diffusion processes, symplectic manifold, integrable family of Hamiltonians, perturbation, averaging principle.

Research benefited from a Royal Society Leverhulme Trust Senior Research Fellowship. 
In the integrable stochastic Hamiltonian system case, the diffusion vector fields span a sub-bundle of the tangent bundle, at least locally. The purpose of the present article is to investigate the effect of a small perturbation to random systems of this type. A solution to an integrable Hamiltonian system preserves the energies $H_{k}$, just as does a solution to any other stochastic Hamiltonian system and there are corresponding invariant manifolds (level sets). The Markov solution restricts to each compact level set and the restriction has generator

$$
\mathcal{L}_{0}=\sum_{k=1}^{n} \frac{1}{2} \mathbf{L}_{X_{H_{k}}} \mathbf{L}_{X_{H_{k}}}+\mathbf{L}_{V}
$$

Here $\mathbf{L}_{V}$ indicates Lie differentiation in the direction of $V$. If the integrable stochastic Hamiltonian system is perturbed by a vector field $\epsilon K$ for $\epsilon>0$ and $K$ a $C^{1}$ vector field not necessarily taking values in the span of $\left\{X_{H_{k}}, k=1,2 \ldots n\right\}$ the solution to the resulting equation

$$
\begin{aligned}
d y_{t}^{\epsilon} & =\sum_{i=1}^{n} X_{H_{k}}\left(y_{t}^{\epsilon}\right) \circ d B_{t}^{k}+V\left(y_{t}^{\epsilon}\right) d t+\epsilon K\left(y_{t}^{\epsilon}\right) d t \\
y_{t}^{\epsilon} & =y_{0}
\end{aligned}
$$

will not conserve the energies. On the other hand letting $\epsilon \rightarrow 0$, the deviation from level sets of the energies will be small. Consider the solution $y^{\epsilon}(t / \epsilon)$ scaled in time by $1 / \epsilon$, which has generator given by $\frac{1}{\epsilon} \mathcal{L}_{0}+\mathbf{L}_{K}$. Note that the motion splits into two parts with the fast component an elliptic diffusion on the invariant torus and the slow motion governed by the transversal part of the vector field $K$. The evolution of $y^{\epsilon}(t / \epsilon)$ is the skew product of the diffusion of order 1 across the level sets and the fast elliptic diffusion of order $\epsilon^{-1}$ along the level sets. The motion on the level sets (thinking of the level sets as the standard n-torus), which would be quasi-periodic if there were no diffusion terms, is ergodic. The evolution of the action component of $y^{\epsilon}(t / \epsilon)$ will not depend on the angular variable in the limit as $\epsilon \rightarrow 0$ and is shown to be described by a system of $n$ ordinary differential equations whose right hand sides can be deduced from $\omega\left(K, X_{H_{i}}\right), i=1, \ldots n$. Here $\omega$ is the symplectic 2-form. The convergence rate is shown to be of order $\epsilon^{\frac{1}{4}}$.

Furthermore if the vector field $K$ is given by a Hamiltonian function, the average of $\omega\left(K, X_{H_{i}}\right)$ over the torus vanishes and we look at the second order scaling to see an interesting limit. The action component of $y^{\epsilon}\left(t \epsilon^{-2}\right)$ involves a martingale term in the limit and the asymptotic law of $y^{\epsilon}\left(t \epsilon^{-2}\right)$ across the level sets is shown to be given by a stochastic differential equation. It remains open to find an estimate for the rate of the convergence of the law of $y^{\epsilon}\left(t \epsilon^{-2}\right)$ to the law of the limiting diffusion.

Main Results. Suppose that $\omega\left(V, X_{H_{i}}\right)=0$ and $V$ commutes with each vector field $X_{H_{i}}$. Let $y_{0} \in M$ be a regular point of $H$ with a neighbourhood $U_{0}$ the domain of an action-angle coordinate map. Let $T^{\epsilon}$ be the first time that the solution $y_{\frac{t}{\epsilon}}$ starting from $y_{0}$ exits $U_{0}$. Set $H^{\epsilon}(t)=\left(H_{1}\left(y_{t / \epsilon}^{\epsilon}\right), \ldots, H_{n}\left(y_{t / \epsilon}^{\epsilon}\right)\right)$. Then $H^{\epsilon}$ converges to the solution of the following system of deterministic equations

$$
\frac{d}{d t} \bar{H}_{i}(t)=\int_{M_{\bar{H}(t)}} \omega\left(X_{H_{i}}, K\right)(\bar{H}(t), z) d \mu_{\bar{H}_{t}}(z),
$$


with corresponding initial condition: If $T^{0}$ is the first time that $\bar{H}(t)$ exits from $U_{0}$ then for all $t<T_{0}, \beta>1$, there exists a constant $C_{2}>0$ such that

$$
\left(\mathbf{E}\left(\sup _{s \leq t}\left\|H^{\epsilon}\left(s \wedge T^{\epsilon}\right)-\bar{H}\left(s \wedge T^{\epsilon}\right)\right\|^{\beta}\right)\right)^{\frac{1}{\beta}} \leq C_{2} \epsilon^{1 / 4}
$$

Furthermore if $r>0$ is such that $U \equiv\left\{x:\left\|H(x)-H\left(y_{0}\right)\right\| \leq r\right\} \subset U_{0}$ define

$$
T_{\delta}=\inf _{t}\left\{\left|\bar{H}_{t}-H\left(y_{0}\right)\right| \geq r-\delta\right\}
$$

Then for any $\beta>1, \delta>0$ and a constant $C$ depending on $T_{\delta}$,

$$
P\left(T^{\epsilon}<T_{\delta}\right) \leq C\left(T_{\delta}\right) \delta^{-\beta} \epsilon^{\beta / 4} .
$$

In the case of $K$ being a smooth local Hamiltonian vector field $\int_{M_{\bar{H}(t)}} \omega\left(X_{H_{i}}, K\right)(\bar{H}(t), z) d \mu_{\bar{H}_{t}}(z)=0$ and hence we could look at the second scaling. The law of the stochastic process $H\left(y_{\frac{t}{2}}^{\epsilon}\right)$ stopped at $S^{\epsilon}$, the first time that the process $y_{\frac{t}{\epsilon^{2}}}^{\epsilon}$ exits from $U_{0}$, converges to that of $H\left(z_{t \wedge S^{\epsilon}}\right)$ where $z_{t}$ is the solution to the following stochastic differential equation

$$
d z_{t}^{j}=\sum_{i} \sigma_{i}^{j}\left(z_{t}\right) \circ d B_{t}^{i}+b_{j}\left(z_{t}\right) d t
$$

Here $\left(\sigma_{i}^{j}\right)$ is the square root of the matrices $\left(a_{i j}\right)$,

$$
a_{i j}(a)=-\int_{M_{a}} \omega\left(K, X_{H_{j}}\right) \mathcal{L}_{0}^{-1}\left(\omega\left(K, X_{H_{i}}\right)\right)(a, z) d \mu_{a}(z)
$$

and

$$
b_{j}(a)=\frac{1}{2} \int_{M_{a}} \mathbf{L}_{K} \mathcal{L}_{0}^{-1}\left(\omega\left(X_{H_{j}}, K\right)\right)(a, z) d \mu_{a}(z) .
$$

We give here a somewhat trivial example of a stochastic integrable system of equations on $\mathbf{R}^{4}$ with the standard symplectic structure:

$$
\begin{aligned}
d x_{1}(t) & =x_{3}(t) d B_{t} \\
d x_{2}(t) & =x_{4}(t) d B_{t}+x_{4} d W_{t} \\
d x_{3}(t) & =-x_{1}(t) d B_{t} \\
d x_{4}(t) & =-x_{2}(t) d B_{t}-x_{2}(t) d W_{t}
\end{aligned}
$$

where $B_{t}$ and $W_{t}$ are independent 1-dimenaional Brownian motions. Take the perturbation vector to be $K_{1}=\left(0, \frac{x_{2}}{x_{2}^{2}+x_{4}^{2}}, 0,0\right), K_{2}=\left(\frac{x_{3}}{\left(x_{1}^{2}+x_{3}^{2}\right)^{2}}, 0, \frac{x_{1}}{\left(x_{1}^{2}+x_{3}^{2}\right)^{2}}, 0\right)$ or $K_{3}=\left(\frac{x_{3}^{2}}{\left(x_{1}^{2}+x_{3}^{2}\right)^{\frac{3}{2}}}, 0,-\frac{x_{1}}{\left(x_{1}^{2}+x_{3}^{2}\right)^{\frac{3}{2}}}, 0\right)$. In the case of $K_{1}$ we have a non-trivial average; in the case of $K_{2}$ and $K_{3}$ we have trivial averages at first scaling and can proceed to the second scaling and obtain a SDE in the limit.

Remark On The Model. This work is in the framework of Arnold on averaging principle of integrable Hamiltonian system as a stochastic Hamiltonian system can be considered as a family of ordinary differential equations with time dependent random vector fields (whose corresponding Hamiltonians are in general neither bounded from below nor differentiable in time). Averaging of stochastic systems was pioneered by Khasminskii [17], Papanicolaou, Stroock and Varadhan [23], and has been an active research field on which there is a rich literature. The structure of the main averaging results are close to that described in the excellent survey of Papanicolaou [22]. We refer to Abraham-Marsden [3], 
Arnold [4], Hofer-Zehnder [16] and McDuff-Salamon [21] as references for Hamiltonian systems on sympletic manifolds, to Givon-Kupferman-Stuart [14] for some physical models behind these problems and for recent progress in the direction of deterministic averaging, to Freidlin-Wentzell [10] and Sowers-Namachchivaya [25] for random perturbations to systems with one degree of freedom, and to Eizenberg-Freidlin [8], BorodinFreidlin [6], Freidlin-Wentzell[11], Sowers [24], Koralov [19], Khasminskii-Krylov [18], and Khasminskii-Yin [20] for recent related work on perturbations of stochastic systems as well as Arnold-Imkeller-Namachchivaya [1] for a discussion on asymptotic expansion of a damped oscillator of one degree of freedom with small noise perturbation. For the Lagrangian mechanics and variational principle in stochastic framework we would like to refer to Bismut's work [5]. However in this article we do not investigate the stochastic mechanics related to the SDEs.

The main novelty of this work is the model itself. We also obtained a rate of convergence. The generator of our perturbed system is:

$$
\mathcal{L}_{0}^{\epsilon}=\sum_{k=1}^{n} \frac{1}{2} \mathbf{L}_{X_{H_{k}}} \mathbf{L}_{X_{H_{k}}}+\mathbf{L}_{V}+\epsilon L_{K}
$$

from which we observe the following aspects of the model: a) The unperturbed random dynamical system is a completely integrable system. b) The fast component of the system is the diffusion term, not the deterministic term. It is also worth noting that the limiting slow motion scaled at $1 / \epsilon$ has $n$ degrees of freedom and is described by a system of $n$ ordinary differential equations. It is only at the second scaling, in the case of the perturbation being Hamiltonian, that we see a limiting $n$-dimensional non-trivial Markov process. The convergence of the slow motion $\left(H_{1}\left(y_{\frac{t}{\epsilon}}^{\epsilon}\right), \ldots, H_{n}\left(y_{\frac{t}{\epsilon}}^{\epsilon}\right)\right)$ is in $L^{p}$ with rate $\epsilon^{\frac{1}{3}}$. At the second scaling the slow motion converges weakly. The assumptions we make on the Hamiltonian functions are: the $\mathbf{R}^{n}$ valued function $\left(H_{1}, \ldots, H_{n}\right)$ is proper and its set of critical points has measure zero.

The following work relates particularly well to ours. We'll point out the differences and similarities. In Dolgopyat [7] the following is proved: If $\hat{h}$ is a first integral of $\dot{y}=$ $\mathbf{E}\left(F\left(y, \xi_{1}\right)\right)$, where $\xi_{1}$ is a random variable of compact support, the piece-wise linear function $h_{\epsilon}$ given by $\hat{h}_{\epsilon}\left(\epsilon^{2} n\right)=\hat{h}\left(x_{n}^{\epsilon}\right)$ converges weakly to the solution of a SDE, under suitable conditions. This was proved using the martingale method. Here $x_{n}^{\epsilon}$ is solution to

$$
x_{n+1}=x_{n}+\epsilon F\left(x_{n}, \xi_{n}\right)+\epsilon^{2} G\left(x_{n}, \xi_{n}\right)+\epsilon^{3} H\left(x_{n}, \xi_{n}, \epsilon\right),
$$

and the $\xi_{n}$ 's are i.i.d. random variables. The equation governing $x_{n}$ are very general. If the perturbation $K$ in (1.2) is given by a Hamiltonian function we may take $F \equiv 0$ in Dolgopyat's model and take $\hat{h}=h$, a first integral to (1.1); however it is not clear how the piece-wise linear function $\hat{h}_{\epsilon}$ relates to the fast motion $h\left(x_{t / \epsilon^{2}}\right)$. In EizenbergFreidlin [8] and Borodin-Freidlin [6], the diffusion part of the motion belongs to the slow component and the fast motion is deterministic. More precisely the perturbed generator is $L_{V}+\epsilon L_{1}+L_{2}$ for a vector field $V$ and an interaction term $L_{2}$. Freidlin and Weber [12] [13] have a different objective. They are mainly concerned with one conserved quantity $H$, not a completely integrable system. The objective there is to obtain a limiting Markov process, using weak convergence, on the graph homeomorphic to the set of connected components of the level sets of $H$. 


\section{Preliminaries}

2.1. Hamiltonian and Symplectic Vector fields. Every symplectic manifold has a natural measure, called the Liouville measure. It is in fact $\wedge^{n} \omega$, differing from the volume form by a constant. Denote by $\iota_{v} \omega$ the inner product of a tangent vector $v$ with $\omega$. The map from $T M$ to $T^{*} M$ given by $v \mapsto \iota_{v} \omega$ is a vector bundle isomorphism, and there is a one to one correspondence between vector fields and differential 1-forms. A symplectic vector field $V$, also called a locally Hamiltonian vector field, is one which preserves the symplectic structure, i.e. $L_{V} \omega=0$. Here $L_{V}$ denotes Lie differentiation in the direction of $V$. Equivalently $\iota_{V} \omega$ is a closed differential 1 -form. For every $C^{1}$ function $H: M \rightarrow \mathbb{R}$ we can associate a Hamiltonian vector field (also called symplectic gradient vector field) given by:

$$
\iota_{X_{H}} \omega=d H .
$$

The canonical sympletic structure on $\mathbf{R}^{2 n}$ with coordinates $\left(q_{1}, \ldots, q_{n}, p_{1}, \ldots, p_{n}\right)$ is $\omega=\sum_{i=1}^{n} d q_{i} \wedge d p_{i}$. Darboux's theorem asserts that any symplectic manifold is locally $\mathbf{R}^{2 n}$ with its canonical sympletic structure. If the first de Rham cohomology $\mathbb{H}^{1}(M ; \mathbf{R})$ vanishes, as in the case of $\mathbf{R}^{2 n}$, every locally Hamiltonian vector field is given by a Hamiltonian function. There are locally Hamiltonian vector fields which are not given by a Hamiltonian function. Take the two torus $T^{2}$ with coordinates $x$ and $y$ periodic in $x$ and $y$. The canonical sympletic structure on $\mathbf{R}^{2}$ induces the symplectic structure on $T^{2}$ : $\omega=d x \wedge d y$. A vector field $X(x, y)=a(x, y) \frac{\partial}{\partial x}+b(x, y) \frac{\partial}{\partial y}$ with $\frac{\partial a}{\partial x}+\frac{\partial b}{\partial y} \equiv 0$ is clearly locally Hamiltonian: the 1-form $\iota_{X} \omega=a(x, y) d y-b(x, y) d x$ is closed. If $a=1, b=0$, the vector field is not given by a Hamiltonian function on $T^{2}$.

The space of smooth functions on $M$ has a Lie algebra structure given by the Poisson bracket. The Poisson bracket of two smooth functions is denoted by $\left\{F_{1}, F_{2}\right\}$ and $\left\{F_{1}, F_{2}\right\}=d F_{1}\left(X_{F_{2}}\right)=\omega\left(X_{F_{1}}, X_{F_{2}}\right)$. The vector field corresponding to the Poisson bracket is precisely the Lie bracket of the Hamiltonian vector fields $X_{F_{1}}$ and $X_{F_{2}}$. Two Hamiltonian functions are Poisson commuting or in involution if their Poisson bracket vanishes, in which case their corresponding Hamiltonian flows commute. If $\{F, H\}=0$ we say that $F$ is a first integral of $H$. Two Hamiltonian functions are said to be linearly independent at $x$ if their associated Hamiltonian vector fields are linearly independent at that point. A family of $n$ Hamiltonian functions is said to form an integrable system if the Hamiltonian functions are pairwise Poisson commuting and if they are linearly independent on a set of full measure.

2.2. An example of a stochastic Hamiltonian system on $\mathbf{R}^{2 n}$. The Hamiltonian vector field given by an Hamiltonian function $H$ is given by $X_{H}=J d H$ where $J$ is the canonical complex structure:

$$
J=\left(\begin{array}{rr}
0 & \mathbf{1} \\
-1 & 0
\end{array}\right)
$$

where 1 denotes the $n$ by $n$ identity matrix. The corresponding Hamiltonian system thus takes the familiar form

$$
\begin{aligned}
\dot{q}_{i}=\frac{\partial H}{\partial p_{i}}, & 1 \leq i \leq n \\
\dot{p}_{i}=-\frac{\partial H}{\partial q_{i}}, & 1 \leq i \leq n .
\end{aligned}
$$

For simplicity write $p=\left(p_{1}, \ldots, p_{n}\right)$ and $q=\left(q_{1}, \ldots, q_{n}\right)$. An important class of examples of Hamiltonian functions on $\mathbf{R}^{2 n}$ is those of the form $H(p, q)=\frac{1}{2}|p|^{2}+V(q)$ for 
some potential function $V$. If $V$ is quadratic, e.g. $V(q)=\frac{1}{2} a^{2}|q|^{2}$, we have the standard harmonic oscillator. The Poisson bracket in $\mathbf{R}^{2 n}$ is of the following form:

$$
\{H, F\}=\sum_{i=1}^{n}\left(\frac{\partial H}{\partial p_{i}} \frac{\partial F}{\partial q_{i}}-\frac{\partial H}{\partial q_{i}} \frac{\partial F}{\partial p_{i}}\right) .
$$

A example of an integrable stochastic Hamiltonian system is given by

$$
\begin{aligned}
d q_{i}(t) & =\frac{\partial K}{\partial p_{i}} d t+\sum_{k=1}^{n} \frac{\partial H_{k}}{\partial p_{i}} \circ d B_{t}^{k} \\
d p_{i}(t) & =-\frac{\partial K}{\partial q_{i}} d t+\sum_{k=1}^{n} \frac{\partial H_{k}}{\partial q_{i}} \circ d B_{t}^{k}
\end{aligned}
$$

where

$$
\begin{aligned}
& H_{1}=\frac{1}{2} \sum_{i=1}^{n} a_{i}^{2} q_{i}^{2}+\frac{1}{2} \sum_{i=1}^{n} p_{i}^{2} \\
& H_{k}=\frac{1}{2} a_{k} q_{k}^{2}+\frac{1}{2} \frac{p_{k}^{2}}{a_{k}}, \quad 2 \leq k<n,
\end{aligned}
$$

and $K$ is a smooth function which commutes with all $H_{i}$ 's, e.g. if $K$ is a smooth function of $H_{i}$ 's.

2.3. The invariant manifolds and integrable symplectic Hamiltonian systems. Let $\left\{H_{k}\right\}_{k=1}^{n}$ be an integrable family of smooth Hamiltonian functions, i.e. they are Poisson commuting and so the $H_{k}$ 's are first integrals of each other and they are independent on a set of full measure. For $a=\left(a_{1}, \ldots, a_{n}\right) \in \mathbf{R}^{n}$ denote by $M_{a}$ the level set of the first integrals $\left\{H_{k}\right\}$ :

$$
M_{a}=\cap_{i=1}^{n}\left\{x: H_{i}(x)=a_{i}\right\} .
$$

The Liouville-Arnold theorem states that if $\left\{H_{k}\right\}_{k=1}^{n}$ are independent on $M_{a}$ then $M_{a}$ is a smooth manifold and furthermore it is diffeomorphic to an $n$ dimensional torus if it is compact and connected. For such value $a, M_{a}$ is invariant under the flows of each $H_{k}$ and each $x$ in $M$ determines an invariant manifold through the value $a=\left(H_{1}(x), \ldots, H_{n}(x)\right)$, which we write also as $M_{H(x)}$.

An application of Itô's formula below shows that the solution flow $\left\{F_{t}(x): t \geq 0\right\}$ of (1.1) preserves the invariant manifolds $\left\{M_{a}\right\}$ :

$$
d H_{i}\left(x_{t}\right)=\sum_{k} d H_{i}\left(X_{H_{k}}\left(x_{t}\right)\right) \circ d B_{t}^{k}+d H_{i}\left(V\left(x_{t}\right)\right) d t=0, \quad 1 \leq i \leq n .
$$

For simplicity we assume throughout the paper the following:

- The invariant manifolds are compact,

which is the case if the map $x \in M \mapsto\left(H_{1}(x), \ldots H_{n}(x)\right) \in \mathbf{R}^{n}$ is proper. Note that the $n$ vector fields $\left\{X_{H_{i}(x)}\right\}$ are tangent to $M_{H(x)}$ and the symplectic form $\omega$ vanishes on the invariant manifolds $M_{a}$. Therefore the stochastic differential equation (1.1) is elliptic when restricted to individual invariant manifolds and the Markovian solution is ergodic. Denote by $\mu_{a}$ the unique invariant probability measure on $M_{a}$; it can be considered as the uniform probability measure on the torus. 
2.4. The invariant measure and th divergence operator for semi-elliptic stochastic symplectic systems. Let $\left\{A^{0}, A^{1}, \ldots, A^{n}\right\}$ be smooth symplectic vector fields with $\left[A^{i}, A^{j}\right]=$ 0 for all $i, j$. Assume that $\left\{A^{1}, \ldots, A^{n}\right\}$ spans a sub-bundle $E$ of the tangent bundle $T M$ of rank $n$. Consider the following stochastic differential equation:

$$
d x_{t}=\sum_{i=1}^{n} A^{i}\left(x_{t}\right) \circ d B_{t}^{i}+A^{0}\left(x_{t}\right) d t .
$$

If there is a global solution flow $\left\{F_{t}\left(x_{0}, \omega\right): t \geq 0\right\}$ to equation (2.1), then the solution flows are stochastic symplectomorphisms, i.e. $\omega=F_{t}^{*} \omega$, where $\omega$ is the symplectic form, not the chance variable.

For each $x \in M$, define a linear map $A(x): \mathbf{R}^{2 n} \rightarrow T_{x} M$ by

$$
A(x)(e)=\sum_{i=1}^{n} A^{i}(x)\left\langle e, e^{i}\right\rangle, \quad e \in \mathbf{R}^{2 n}
$$

where $\left\{e^{i}\right\}$ is an orthonormal basis of $\mathbf{R}^{2 n}$. The linear map is onto $E_{x}$ with kernel $\{0\} \times$ $\mathbf{R}^{n}$ and gives a positive symmetric bilinear form on $E$ by making $\left\{A^{i}(x)\right\}$ an orthonormal basis:

$$
\left\langle A^{i}(x), A^{j}(x)\right\rangle=\delta_{i j} .
$$

Then $A(x)$ is an isomorphism from $\mathbf{R}^{n} \times\{0\}$ to $E_{x}$. This defines a metric on $E$ : for $u=\sum_{i} u_{i} A^{i}$ and $v=\sum_{i} v_{i} A^{i}$,

$$
\langle u, v\rangle=\sum_{i=1}^{n} u_{i} v_{i}
$$

and for a function $f$ we define its gradient $\nabla_{E} f=\sum_{i} d f\left(A^{i}\right) A^{i}$. The symplectic structure $\omega$ restricts to $E$ defines a complex structure on $M$ as following: we first give the tangent bundle $T M$ any Riemannian metric which agrees with the one constructed on $E$ using the linear map $A$. Define $J_{x}: T_{x} M \rightarrow T_{x} M$ by

$$
\omega\left(J_{x} u, v\right)=\langle u, v\rangle_{x} .
$$

To see that this identity defines $J_{x}$ uniquely suppose that for $u \in T_{x} M$ there are $u_{1}$ and $u_{2}$ satisfies $\omega\left(u_{i}, v\right)=\left\langle u_{i}, v\right\rangle_{x}, i=1,2$. Then $\omega\left(u_{1}-u_{2}, v\right)=0$ for all $v$. Thus $u_{1}=u_{2}$. Existence can be easily seen as direct calculations can be done in $\mathbf{R}^{2 n}$.

Next take $A^{i}=X_{H_{i}}$ in (2.1), $i=1,2, \ldots n$, to be the Hamiltonian vector fields for an integrable family of Hamiltonian functions $\left\{H_{i}\right\}$ and $A^{0}=V$. We arrive back to the integrable stochastic sympletic equation (1.1) where $V$ is a symplectic vector field commuting with all $X_{H_{i}}$ 's. Under our assumption that

$$
H: x \rightarrow\left(H_{1}(x), \ldots H_{n}(x)\right)
$$

is a proper map, then for almost every point $a_{0}$ in $\mathbf{R}^{n}$ it is either trvial or a local trivial fibration in the sense that there is a neighbourhood $V$ of $a_{0}$ such that $H^{-1}(a)$ is a smooth sub-manifold for all $a \in V$ and that there is a diffeomorphism from $H^{-1}(V)$ to $V \times$ $H^{-1}\left(a_{0}\right)$. Such $a_{0}$ is called a regular value of $H$. Denote by $\Sigma_{H}$ the set of values in $\mathbf{R}^{n}$ which are not regular. A point $y$ in $M$ is said to be a critical point if $H(y) \in \Sigma_{H}$. By Sard's theorem the set of critical values of the function $H$ has measure zero. The $2 n$-differential form $\omega^{n}$, as a measure, has a decomposition which gives a measure on each invariant manifold $M_{a}$ for regular $a$ value. The decomposition can be chosen in the following way. First recall that on a neighbourhood of a regular point $a_{0}$ of $H$, every component of the level set $M_{a_{0}}$ is diffeomorphic to an $n$-torus and a small neighbourhood $U_{0}$ of $M_{a_{0}}$ is 
diffeomorphic to the product space $T^{n} \times D$ where $D$ is a relatively compact open set in $\mathbf{R}^{n}$, see e.g. [2]. More precisely if $V$ is an open set of $\mathbf{R}^{n}$ such that $H^{-1}(V)$ does not contain any critical points of $H$ then it is diffeomorphic to $D \times T^{n}$. Take an action angle chart around $M_{a}$ which is diffeomorphic to $D \times T^{n}$ for some open set $D$. The measure $\left(\sum_{i} d I^{i} \wedge d \theta^{i}\right)^{n}$ on the product space naturally splits to give us a probability measure, the Haar measure $d \theta^{1} \wedge \ldots d \theta^{n}$ on $T^{n}$. We take the corresponding one on $M_{a}$ and denote it by $\mu_{a}$. Let $U$ be a section of $E$. Define the divergences $\operatorname{div}_{E}^{a} U$ to be the functions such that

$$
\int_{M_{a}} d f(U) d \mu_{a}=-\int_{M_{a}} f \operatorname{div}_{E}^{a} U d \mu_{a}
$$

for all smooth functions $f$ on $M_{a}$. Note that $\operatorname{div}_{E} X_{H_{i}}=0$, since

$$
\int_{M_{a}} d f\left(X_{H_{i}}\right) d \mu_{a}=\int_{M_{a}}\left\{H_{i}, f\right\} d \mu_{a}=0
$$

for all smooth functions $f$ (see the beginning of section 4 for a calculation). Thus if $U=$ $\sum_{i} a_{i} X_{H_{i}}$ where $a_{i}$ are constant on $M_{a}$ and is thus divergence free.

Remark 2.1. Let $U$ be a section of $E$ commuting with all $X_{H_{i}}$ the invariant measure for the SDE (1.1) restricted to the invariant manifold $M_{a}$ is $\mu_{a}$, which varies smoothly with $a$ in sufficiently small neighbourhoods of a regular value.

Proof. The measure $\omega^{n}$ is an invariant measure for the SDE on $M$ due to the fact that the solution of the SDE leaves invariant the symplectic form. More precisely, since $U$ commutes with $\left\{X_{H_{i}}\right\}$ and thus can be written in the the form of $U=\sum_{i} a_{i} X_{H_{i}}(x)$, where $a_{i}$ are constant on $M_{a}$, it is therefore divergence free. Thus the invariant measure of the SDE restricted to the torus is the same as that of the corresponding SDE without a drift. From the action angle transformation we see that the measure $\mu_{a}$ is an invariant measure for the SDE restricted to $M_{a}$. This is in fact the only invariant measure for the SDE on $M_{a}$ since the system is elliptic when restricted to each level set and the conclusion follows.

\section{An Averaging Principle}

Let $\left\{H_{i}\right\}_{i=1}^{n}$ be a completely integrable system on a smooth $2 n$-dimensional symplectic manifold $M$ so that the functions $\left\{H_{i}\right\}$ are constants of motions of each other and that they are pairwise in involution. We assume that the $\mathbf{R}^{n}$-valued function $H=\left(H_{1}, \ldots, H_{n}\right)$ is proper and its set of critical points has measure zero. Note that the vector fields $\left\{X_{H_{i}}\right\}$ form an integrable distribution and through each point of the manifold there is an integrable $n$ dimensional manifold.

Take an action-angle coordinate: $\phi^{-1}: U_{0} \rightarrow D \times T^{n}$. In this coordinate, $x=\phi(I, \theta)$, $I \in D, \theta \in T^{n}$, and $\left(\phi^{-1}\right)_{*} \omega=d I \wedge d \theta$ defines a symplectic structure on $D \times T^{n}$. Furthermore if $\tilde{H}_{i}=H_{i}(\phi(I, \theta))$ is the induced Hamiltonian on $D \times T^{n}$ then $\dot{I}_{i}^{k}=$ $-\frac{\partial \tilde{H}_{i}}{\partial \theta_{k}}=0$ and

$$
\dot{\theta}_{i}^{k}=\frac{\partial \tilde{H}_{i}}{\partial I_{k}}=\omega_{i}^{k}(I)
$$

with $\omega_{i}^{k}$ smooth functions. In fact $X_{\tilde{H}_{i}}=\left(\phi^{-1}\right)_{*}\left(X_{H_{i}}\right)=-\sum_{k=1}^{n} \frac{\partial\left(H_{i} \circ \phi\right)}{\partial I_{k}} \frac{\partial}{\partial \theta_{k}}$. For example the integrable Hamiltonian system in section 2.2 is equivalent to the Hamiltonian system $H_{1}=\sum_{i=1}^{n} a_{i} \bar{q}_{i}, a_{i}>0$, and $H_{k}=\bar{q}_{k}, k=2, \ldots, n$, through the action angle 
coordinates change $(q, p) \mapsto(\bar{q}, \bar{p})$ :

$$
\begin{aligned}
& \left(q_{1}, \ldots q_{n}, p_{1}, \ldots, p_{n}\right) \\
= & \left(\sqrt{\frac{2 \bar{q}_{1}}{a_{1}}} \cos \bar{p}_{1}, \ldots \sqrt{\frac{2 \bar{q}_{n}}{a_{n}}} \cos \bar{p}_{n}, \sqrt{2 a_{1} \bar{q}_{1}} \sin \bar{p}_{1}, \ldots, \sqrt{2 a_{n} \bar{q}_{n}} \sin \bar{p}_{n},\right) .
\end{aligned}
$$

The corresponding Hamiltonian system is the trivial one $\dot{p}_{i}=a_{i}, \dot{q}_{i}=0$. Since $U_{0}$ is diffeomorphic to $D \times T^{n}$ there is a constant $r>0$ such that $U_{0}$ contains the open set $\left\{x: \sum_{i}\left|H_{i}(x)-H_{i}\left(y_{0}\right)\right|^{2} \leq r^{2}\right\}$.

Let $K$ be a smooth vector field, transversal in the sense that $\omega\left(X_{H_{i}}, K\right)$ are not all identically zero. Denote by $y_{t}^{\epsilon}$ the solution to (1.2), the perturbation of the integrable system (1.1) starting from a given point $y_{0}$ in $M$. Set $x_{t}=y_{t}^{0}$, the solution to (1.1) with initial value $y_{0}$. If $V$ is a vector field on $M$ denote by $\tilde{V}$ the induced vector field on $D \times T^{n}$. We assume the following of the SDE (1.2):

Condition R: Suppose that $\omega\left(V, X_{H_{i}}\right)=0$ and $V$ commutes with all vector fields $X_{H_{i}}$. Let $y_{0} \in M$ be a regular point of $H$ with a neighbourhood $U_{0}$ the domain of an actionangle coordinate map: $\phi^{-1}: U_{0} \rightarrow D \times T^{n}$, where $D$ is an open set of $\mathbf{R}^{n}$.

We adopt the following notation: if $f$ is a function on $U_{0}$, by $\tilde{f}$ we mean the representation of $f$ in $D \times T^{n}$.

Lemma 3.1. Assume condition $R$ holds for (1.2). Let $\tau^{\epsilon}$ be the first time that the solution $y_{t}^{\epsilon}$ starting from $y_{0}$ exits $U_{0}$. Then for any smooth function $f$ on $M$,

(1)

$$
\left[\mathbf{E}\left(\sup _{s \leq t \wedge \tau^{\epsilon}}\left|f\left(y_{s}^{\epsilon}\right)-f\left(x_{s}\right)\right|^{p}\right)\right]^{\frac{1}{p}} \leq C_{1} \epsilon\left(t+t^{2}\right),
$$

where $C_{1}=C_{1}\left(V, K, H_{i}, f\right)$ depends on the upper bounds of the functions $|d \tilde{f}|$, $\left|\frac{\partial^{2} \tilde{H}_{k}}{\partial I_{i} \partial I_{j}}\right|,|d \tilde{V}|,|\tilde{K}|$ on $D \times T^{n}$.

(2) If $V \equiv 0$, then the estimates above, $\epsilon\left(t+t^{2}\right)$, can be improved to $C_{1} \epsilon\left(t+t^{\frac{3}{2}}\right)$.

Proof. In the proof below $C$ stands for an unspecified constant. We denote the flows in action-angle coordinates by $x_{t}=\phi\left(I_{t}, \theta_{t}\right)$ and $y_{t}^{\epsilon}=\phi\left(I_{t}^{\epsilon}, \theta_{t}^{\epsilon}\right)$. Set $\tilde{f}=f \circ \phi$. Then

$$
\begin{aligned}
\left|f\left(y_{t}^{\epsilon}\right)-f\left(x_{t}\right)\right| & =\left|\tilde{f}\left(I\left(y_{t}^{\epsilon}\right), \theta\left(y_{t}^{\epsilon}\right)\right)-\tilde{f}\left(I\left(x_{t}\right), \theta\left(x_{t}\right)\right)\right| \\
& \leq C\left|I\left(y_{t}^{\epsilon}\right)-I\left(x_{t}\right)\right|+C\left|\theta\left(y_{t}^{\epsilon}\right)-\theta\left(x_{t}\right)\right|,
\end{aligned}
$$

using the fact that $\frac{\partial \tilde{f}}{\partial I}$ and $\frac{\partial \tilde{f}}{\partial \theta}$ are bounded on $T^{n} \times D$ as $D$ is relatively compact. In the local chart, $\frac{\partial \tilde{V}}{\partial \theta_{i}}=0$ and we can write $V(I, \theta)=V_{j}(I) \frac{\partial H_{j}}{\partial I}(I, \theta)=\omega_{0}^{j}(I) \frac{\partial}{\partial \theta_{j}}$ for some smooth functions $\omega_{0}^{j}$ on $D$. The perturbation vector field can be written as $\left(K^{\theta}, K^{I}\right)$ where $K_{\theta}=\left(K_{\theta}^{1}, \ldots, K_{\theta}^{n}\right)$ and $K_{I}=\left(K_{I}^{1}, \ldots, K_{I}^{n}\right)$ be respectively the angle and the action component of the vector field $\tilde{K}$ on $T^{n} \times D^{n}$. The result is now clear from the form of the SDE on $T^{n} \times D$ :

$$
\begin{aligned}
d I_{t}^{\epsilon, i} & =\epsilon K_{I}^{i}\left(I_{t}^{\epsilon}, \theta_{t}^{\epsilon}\right) d t \\
d \theta_{t}^{\epsilon, i} & =\sum_{k=1}^{n} \omega_{k}^{i}\left(I_{t}^{\epsilon}\right) \circ d B_{t}^{k}+\omega_{0}^{i}\left(I_{t}^{\epsilon}\right) d t+\epsilon K_{\theta}^{i}\left(I_{t}^{\epsilon}, \theta_{t}^{\epsilon}\right) d t
\end{aligned}
$$


where $\omega_{k}^{i}, i, k=1, \ldots n$, are defined by (3.1). Indeed, then

$$
\sup _{s \leq t \wedge \tau^{\epsilon}}\left|I_{s}^{\epsilon}-I_{s}\right|=\epsilon \sup _{s \leq t \wedge \tau^{\epsilon}}\left|\int_{0}^{s}\right| K_{I}\left(I_{s}^{\epsilon}, \theta_{I}^{\theta}\right)\left|d s \leq \epsilon t \sup _{D \times T^{n}}\right| K_{I} \mid,
$$

and for $s<\tau^{\epsilon}$,

$$
\begin{aligned}
\theta_{s}^{\epsilon, i}-\theta_{s}^{i}= & \sum_{k=1}^{n} \int_{0}^{s}\left(\omega_{k}^{i}\left(I_{r}^{\epsilon}\right)-\omega_{i}^{k}\left(I_{r}\right)\right) \circ d B_{r}^{k} \\
& +\int_{0}^{s}\left(\omega_{0}^{i}\left(I_{r}^{\epsilon}\right)-\omega_{0}^{i}\left(I_{r}\right)\right) d r+\epsilon \int_{0}^{s} K_{\theta}^{i}\left(I_{r}^{\epsilon}, \theta_{r}^{\epsilon}\right) d r .
\end{aligned}
$$

As the Stratonovitch correction for the SDE vanishes, we may replace the Stratonovitch integration by Itô integration:

$$
\int_{0}^{s}\left(\omega_{k}^{i}\left(I_{r}^{\epsilon}\right)-\omega_{k}^{i}\left(I_{r}\right)\right) \circ d B_{r}^{k}=\int_{0}^{s}\left(\omega_{k}^{i}\left(I_{r}^{\epsilon}\right)-\omega_{k}^{i}\left(I_{r}\right)\right) d B_{r}^{k}
$$

Consequently,

$$
\begin{aligned}
\left|\theta_{i}\left(y_{s}^{\epsilon}\right)-\theta_{i}\left(x_{s}\right)\right| \leq & \left|\sum_{k=1}^{n} \int_{0}^{s}\left(\omega_{k}^{i}\left(I_{r}^{\epsilon}\right)-\omega_{i}^{k}\left(I_{r}\right)\right) d B_{r}^{k}\right| \\
& +\sup _{D \times T^{n}}\left|d \omega_{0}^{i}\right| \cdot \int_{0}^{s}\left|I_{r}^{\epsilon}-I_{r}\right| d r+\epsilon s \sup _{D \times T^{n}}\left|K_{\theta}^{i}\right| \\
\leq & \left|\sum_{k=1}^{n} \int_{0}^{s}\left(\omega_{k}^{i}\left(I_{r}^{\epsilon}\right)-\omega_{i}^{k}\left(I_{r}\right)\right) d B_{r}^{k}\right| \\
& +\epsilon \frac{s^{2}}{2} \sup _{D \times T^{n}}\left|K_{I}\right| \cdot \sup _{D \times T^{n}}\left|d \omega_{i}^{0}\right|+\epsilon s \sup _{D \times T^{n}}\left|K_{\theta}^{i}\right| .
\end{aligned}
$$

Summing up over $i$, we have

$$
\begin{aligned}
& \mathbf{E} \sup _{s \leq t \wedge \tau^{\epsilon}}\left|\theta_{s}^{\epsilon}-\theta_{s}\right|^{p} \\
\leq & C_{1} \sup _{s \leq t}\left(\sum_{i, k=1}^{n} \mathbf{E} \int_{0}^{s}\left|\omega_{k}^{i}\left(I_{r}^{\epsilon}\right)-\omega_{k}^{i}\left(I_{r}\right)\right|^{2}\right)^{p / 2}+C_{2}(\tilde{K}) \epsilon\left(t+t^{2}\right)^{p} \\
\leq & C_{1}\left(\sum_{i, k}\left(\left|d \omega_{k}^{i}\right| \vee 1\right)^{p}\right) \epsilon^{p} t^{\frac{3 p}{2}}+C_{2}(\tilde{K}) \epsilon^{p}\left(t+t^{2}\right)^{p}
\end{aligned}
$$

by $L_{p}$ inequalities for martingales. Combining the estimates we obtain

$$
\mathbf{E} \sup _{s \leq t \wedge \tau^{\epsilon}}\left|f\left(y_{s}^{\epsilon}\right)-f\left(x_{s}\right)\right| \leq C_{3} \epsilon\left(t+t^{2}\right)
$$

for some constant $C_{3}$.

(ii) If the drift $V \equiv 0$ then $\omega_{0}=0$ and the calculation above shows that the estimate is of the order $\epsilon\left(t^{\frac{3}{2}} \wedge 1\right)$.

If the stochastic dynamical system (1.1) is subjected to a small non-Hamiltonian perturbation, the slow variable is in the direction transversal to the energy surfaces while the stochastic components are the fast variables. The lemma shows that the first integrals of the perturbed system change by an order $\epsilon\left(t+t^{2}\right)$ over a time interval $t$ and so the slow component accumulates over a time interval of the size $t / \epsilon$ and we obtain a new dynamical system in the limit: as $\epsilon$ goes to zero the motion along the torus is significantly faster compared to the motion in the transversal direction and thus the action component of $y_{t / \epsilon}^{\epsilon}$ has 
a limit as the randomness in the fast component is averaged out by the induced invariant measure, as shown below. Recall that $H(x)=\left(H_{1}(x), \ldots, H_{n}(x)\right)$.

We first prove a lemma:

Lemma 3.2. Assume condition $R$ holds. Let $g$ be a real-valued function on $M$, which is considered in the action angle co-ordinates as a function from $D \times T^{n}$ to $\mathbf{R}$. Define $Q^{g}: D \subset \mathbf{R}^{n} \rightarrow \mathbf{R}$ to be its average over the torus:

$$
Q^{g}(a)=\int_{T^{n}} \tilde{g}(a, z) d \mu(z) .
$$

Suppose that $g$ is $C^{1}$ on $U_{0}$. Set

$$
H_{i}^{\epsilon}(s)=H_{i}\left(y_{s / \epsilon}^{\epsilon}\right), \quad H^{\epsilon}(s)=\left(H_{1}^{\epsilon}(s), \ldots, H_{n}^{\epsilon}(s)\right) .
$$

Then

$$
\int_{s \wedge T^{\epsilon}}^{(s+t) \wedge T^{\epsilon}} g\left(y_{r / \epsilon}^{\epsilon}\right) d r=\int_{s \wedge \tau^{\epsilon}}^{(s+t) \wedge \tau^{\epsilon}} Q^{g}\left(H^{\epsilon}(r)\right) d r+\delta(g, \epsilon, t)
$$

with the following rate of convergence: for any $\beta>1$,

$$
\left(\mathbf{E} \sup _{s \leq t}|\delta(g, \epsilon, s)|^{\beta}\right)^{\frac{1}{\beta}} \leq C(t) \epsilon^{\frac{1}{4}} .
$$

where $T^{\epsilon}$ is the first time that $y_{t / \epsilon}^{\epsilon}$ exits from $U_{0}$ and $\tau^{\epsilon}=T^{\epsilon} / \epsilon$.

Proof. The idea is to approximate $g\left(y_{r}^{\epsilon}\right)$ by $g\left(y_{r}\right)$ on sufficiently small intervals and to apply the law of large numbers to each integral bearing in mind that the cost to replace $y_{r}^{\epsilon}$ by $y_{r}$ is of order $\epsilon \delta$ on an interval of size $\delta$, assuming that $\delta \rightarrow \infty$ as $\epsilon \rightarrow 0$, and that the error term for replacing time average by space average is of order $\frac{1}{\sqrt{\delta}}$.

Let $\tau^{\epsilon}$ be the first time that $y_{t}^{\epsilon}$ exits from $U_{0}$. For $q \in(0,1)$, let $\Delta t=\frac{(t+s)}{\epsilon^{q}} \wedge \tau^{\epsilon}-\frac{s}{\epsilon^{q}} \wedge \tau^{\epsilon}$, which is of order $\epsilon^{-q}$, and set $N \equiv N(\epsilon)=\left[\epsilon^{q-1}\right]+1$ which is of order $\epsilon^{q-1}$. Here $\left[\epsilon^{q-1}\right]$ is the integer part of $\epsilon^{q-1}$ and all terms may depend on the sample paths of $\omega$. Take $t_{n}=\frac{s}{\epsilon} \wedge \tau^{\epsilon}+n \Delta t, 1 \leq n \leq N-1$, so that

$$
\frac{s}{\epsilon} \wedge \tau^{\epsilon}=t_{0}<t_{1}<\cdots<t_{N-1}<\frac{s+t}{\epsilon} \wedge \tau^{\epsilon} .
$$

We first make some pathwise estimates. For any $C^{1}$ function $g$ on $M$,

$$
\begin{aligned}
\left|\int_{s \wedge T^{\epsilon}}^{(s+t) \wedge T^{\epsilon}} g\left(y_{u / \epsilon}^{\epsilon}\right) d u\right| & =\left|\epsilon \int_{\frac{s}{\epsilon} \wedge \tau^{\epsilon}}^{\frac{s+t}{\epsilon} \wedge \tau^{\epsilon}} g\left(y_{r}^{\epsilon}\right) d r\right| \\
& \leq \epsilon\left|\sum_{n=0}^{N-1} \int_{t_{n}}^{t_{n+1}} g\left(y_{r}^{\epsilon}\right) d r\right|+\epsilon\left|\int_{t_{N}}^{\frac{s+t}{\epsilon} \wedge \tau^{\epsilon}}\right| g\left(y_{r}^{\epsilon}\right) d r
\end{aligned}
$$

Since $g$ is bounded on $U_{0}$, the second term on the right hand side of the above expression converges to zero with rate $\epsilon^{1-q}$ :

$$
\epsilon\left|\int_{t_{N}}^{\frac{(s+t)}{\epsilon} \wedge \tau^{\epsilon}} g\left(y_{r}^{\epsilon}\right) d r\right| \leq C \epsilon \Delta t \leq C t \epsilon^{1-q} .
$$


For the remaining terms we use the splitting

$$
\begin{aligned}
\epsilon \sum_{n=0}^{N-1} \int_{t_{n}}^{t_{n+1}} g\left(y_{r}^{\epsilon}\right) d r= & \epsilon \sum_{n=0}^{N-1} \int_{t_{n}}^{t_{n+1}}\left[g\left(y_{r}^{\epsilon}\right)-g\left(F_{r-t_{n}}\left(y_{t_{n}}^{\epsilon}, \Theta_{t_{n}}(\omega)\right)\right)\right] d r \\
& +\epsilon \sum_{n=0}^{N-1} \int_{t_{n}}^{t_{n+1}} g\left(F_{r-t_{n}}\left(y_{t_{n}}^{\epsilon}, \Theta_{t_{n}}(\omega)\right)\right) d r .
\end{aligned}
$$

where $\omega$ denotes the chance variable, $\Theta_{t}$ the shift operator on the canonical probability space: $\Theta_{t}(\omega)(-)=\omega(-+t)-\omega(t)$, and $\left\{F_{t}(x, \omega), t \geq 0\right\}$ the solution flow of the unperturbed stochastic differential equation (1.1) with starting point $x$. Write the summation as the sum of $A_{1}$ and $A_{2}$ and the second term is:

$$
A_{2}(t, \epsilon) \equiv \epsilon \sum_{n=0}^{N-1} \int_{t_{n}}^{t_{n+1}} g\left(F_{r-t_{n}}\left(y_{t_{n}}^{\epsilon}, \Theta_{t_{n}}(\omega)\right)\right) d r .
$$

The law of the large numbers says that for any function $f$ on a compact manifold converging to infinity as $t$ converges to infinity, $\frac{1}{t} \int_{s}^{s+t} f\left(x_{r}\right) d r$ converges to $\int_{M} f(z) d z$ when $t \rightarrow \infty$ with rate $\frac{1}{\sqrt{t}}$ and the convergence is uniform on compact time intervals in $L^{p}$ for all $p>1$. Here $d z$ is the volume measure. The easiest way to see that this holds is to first assume that $\int f d z$ vanishes and let $h$ be the function solving $\Delta h=2 f$ and apply Itô's formula to $h\left(x_{t}\right)$ on the time interval $[s, s+t]$. Note that the intervals $\left[t_{i}, t_{i+1}\right]$ are either constant intervals or intervals of zero length with the exception of one bad interval of the form $\left[a, \tau^{\epsilon}\right]$ of size at most $\Delta t$. Let $M$ be the integer such that $\left[t_{i}, t_{i+1}\right]$ are constant intervals for $i<M$. For the bad interval $\left[t_{M}, \tau^{\epsilon}\right)$, the following term in $A_{2}$

$$
\epsilon \int_{t_{M}}^{\tau^{\epsilon}} g\left(F_{r-t_{n}}\left(y_{t_{n}}^{\epsilon}, \Theta_{t_{n}}(\omega)\right)\right) d r
$$

is of order $\epsilon \Delta t$. On each constant interval $\left[t_{i}, t_{i+1}\right], i<M$, the corresponding term in $A_{2}$ is:

$$
\epsilon \int_{t_{n}}^{t_{n+1}} g\left(F_{r-t_{n}}\left(y_{t_{n}}^{\epsilon}, \Theta_{t_{n}}(\omega)\right)\right) d r=\epsilon \int_{0}^{\Delta t} g\left(F_{r}\left(y_{t_{n}}^{\epsilon}, \Theta_{t_{n}}(\omega)\right)\right) d r .
$$

Denote by $\mu_{H^{\epsilon}\left(\epsilon t_{n}\right)}$, or $\mu_{y_{t_{n}}^{\epsilon}}$, the invariant measure on the invariant manifold $M_{H^{\epsilon}\left(\epsilon t_{n}\right)} \equiv$ $M_{y_{t_{n}}^{\epsilon}}$. We apply the law of large numbers to such terms and use the Markov property of the flow to obtain the following estimates, for all sufficiently small $\epsilon$,

$$
\begin{aligned}
& {\left[\mathbf{E} \sup _{u \leq t}\left(A_{2}(u, \epsilon)-\epsilon \Delta t \sum_{n=0}^{N-1} \int_{M_{H^{\epsilon}\left(\epsilon t_{n}\right)}} g\left(H^{\epsilon}\left(\epsilon t_{n}\right), z\right) d \mu_{H^{\epsilon}\left(\epsilon t_{n}\right)}(z)\right)^{\beta}\right]^{\frac{1}{\beta}}} \\
& \leq C \epsilon \Delta t+ \\
& N \sup _{n}\left[\mathbf{E}\left(\epsilon \int_{0}^{\Delta t} g\left(F_{r}\left(y_{t_{n}}^{\epsilon}, \Theta_{t_{n}}(\omega)\right)\right) d r-\epsilon \Delta t \int_{M_{H^{\epsilon}\left(\epsilon t_{n}\right)}} g\left(H^{\epsilon}\left(\epsilon t_{n}\right), z\right) d \mu_{H^{\epsilon}\left(\epsilon t_{n}\right)}(z)\right)^{\beta}\right]^{\frac{1}{\beta}} \\
& \leq C \epsilon \Delta t+ \\
& (\epsilon \Delta t) N \sup _{n}\left(\mathbf{E} \sup _{u \leq t}\left|\frac{1}{\Delta t} \int_{0}^{\Delta t} g\left(F_{r}\left(y_{t_{n}}^{\epsilon}, \Theta_{t_{n}}(\omega)\right)\right) d r-\int_{M_{H^{\epsilon}\left(\epsilon t_{n}\right)}} g\left(H^{\epsilon}\left(\epsilon t_{n}\right), z\right) d \mu_{H^{\epsilon}\left(\epsilon t_{n}\right)}(z)\right|^{\beta}\right)^{\frac{1}{\beta}} \\
& \leq C t \epsilon^{1-q}+C \frac{\epsilon \Delta t N}{\sqrt{t / \epsilon^{q}}} \leq C t \epsilon^{1-q}+C \sqrt{t} \epsilon^{\frac{q}{2}}
\end{aligned}
$$


AN AVERAGING PRINCIPLE FOR A COMPLETELY INTEGRABLE STOCHASTIC HAMILTONIAN SYSTEM 13

On the other hand letting $s_{n}=\epsilon t_{n}$ so $\Delta s=\epsilon \Delta t$ and consider $s_{0}<s_{1} \cdots<s_{N}$. As $\Delta s$ is of order $O\left(\epsilon^{1-q}\right)$, the following pathwise estimate follows:

$$
\begin{aligned}
& \left|\Delta s \sum_{n=0}^{N-1} \int_{M_{H^{\epsilon}\left(s_{n}\right)}} g\left(H^{\epsilon}\left(s_{n}\right), z\right) d \mu_{H^{\epsilon}\left(s_{n}\right)}(z)-\int_{s \wedge \tau^{\epsilon}}^{(s+t) \wedge \tau^{\epsilon}} \int_{M_{H^{\epsilon}(s)}} g\left(H^{\epsilon}\left(s_{n}\right), z\right) d \mu_{H^{\epsilon}(r)}(z) d r\right| \\
& \leq C(g) t \epsilon^{1-q}
\end{aligned}
$$

where $C(g)=\max _{U_{0}}|d g|$. To summarise:

$$
\int_{s \wedge \tau^{\epsilon}}^{(s+t) \wedge \tau^{\epsilon}} g\left(y_{r / \epsilon}^{\epsilon}\right) d r=\int_{s \wedge \tau^{\epsilon}}^{(s+t) \wedge \tau^{\epsilon}} Q^{g}\left(H^{\epsilon}(r)\right) d r+\delta(g, \epsilon, t)
$$

where

$$
\begin{aligned}
& |\delta(g, \epsilon, t)| \\
\leq & C \epsilon^{1-q}+\left|\epsilon \int_{t_{N}}^{\frac{(t+s)}{\epsilon} \wedge \tau^{\epsilon}} g\left(y_{r}^{\epsilon}\right) d r\right|+\left|A_{1}(t, \epsilon)\right|+ \\
& \left|A_{2}(t, \epsilon)-\sum \epsilon \Delta t \int_{M_{H^{\epsilon}\left(\epsilon t_{n}\right)}} g\left(H^{\epsilon}\left(\epsilon t_{n}\right), z\right) d \mu_{H^{\epsilon}\left(\epsilon t_{n}\right)}(z)\right| \\
& +\left|\sum \Delta s \int_{M_{H^{\epsilon}\left(s_{n}\right)}} g\left(H^{\epsilon}\left(s_{n}\right), z\right) d \mu_{H^{\epsilon}\left(s_{n}\right)}(z)-\int_{s \wedge \tau^{\epsilon}}^{(s+t) \wedge \tau^{\epsilon}} \int_{M_{H^{\epsilon}(s)}} g\left(H^{\epsilon}(s), z\right) d \mu_{H^{\epsilon}(s)}(z) d s\right|
\end{aligned}
$$

and

$$
A_{1}(t, \epsilon)=\epsilon \sum_{n=0}^{N-1} \int_{t_{n}}^{t_{n+1}}\left[g\left(y_{r}^{\epsilon}\right)-g\left(F_{r-t_{n}}\left(y_{t_{n}}^{\epsilon}, \Theta_{t_{n}}(\omega)\right)\right)\right] d r .
$$

By the previous estimates:

$$
|\delta(g, \epsilon, t)| \leq C t \epsilon^{1-q}+C t^{\frac{1}{2}} \epsilon^{q / 2}+\left|A_{1}(t, \epsilon)\right| .
$$

To show that $\left|A_{1}\right|$ is reasonably small, we use Kolmogorov's theorem, apply Lemma 3.1 and Hölder's inequality

$$
\begin{aligned}
& \left(\mathbf{E} \sup _{s \leq t}\left(A_{1}(s, \epsilon)\right)^{\beta}\right)^{\frac{1}{\beta}} \\
\leq & C t \epsilon^{1-q}+\epsilon\left[\mathbf{E} \sup _{s \leq t}\left(\sum_{n=0}^{N-1} \int_{t_{n}}^{t_{n+1}}\left|g\left(y_{r}^{\epsilon}\right)-g\left(F_{r-t_{n}}\left(y_{t_{n}}^{\epsilon}, \Theta_{t_{n}}(\omega)\right)\right)\right| d r\right)^{\beta}\right]^{\frac{1}{\beta}} \\
\leq & C t \epsilon^{1-q}+\epsilon \cdot N^{1-1 / \beta}\left(\sum_{n=0}^{N-1} \mathbf{E}\left[\sup _{s \leq t} \int_{t_{n}}^{t_{n+1}}\left|g\left(y_{r}^{\epsilon}\right)-g\left(F_{r-t_{n}}\left(y_{t_{n}}^{\epsilon}, \Theta_{t_{n}}(\omega)\right)\right)\right| d r\right]^{\beta}\right)^{\frac{1}{\beta}} \\
\leq & C t \epsilon^{1-q}+\epsilon N^{1-1 / \beta} \cdot N^{\frac{1}{\beta}} C \cdot \epsilon\left(\Delta t+(\Delta t)^{2}\right) \Delta t \\
\leq & C t^{2} \epsilon^{1-2 q}+C t \epsilon^{1-q} .
\end{aligned}
$$

Consequently,

$$
\left(\mathbf{E} \sup _{s \leq t}|\delta(g, \epsilon, s)|^{\beta}\right)^{\frac{1}{\beta}} \leq C t^{2} \epsilon^{1-2 q}+C t^{\frac{1}{2}} \epsilon^{q / 2}+C t \epsilon^{1-q}
$$

and finally take $q=1 / 4$ to obtain:

$$
\left\|\sup _{s \leq t} \delta(g, \epsilon, s)\right\|_{L_{\beta}} \leq C t^{2} \epsilon^{\frac{1}{4}}+C \epsilon^{\frac{1}{4}} t^{\frac{1}{2}} .
$$


Theorem 3.3. Consider the stochastic differential equation (1.2) satisfying condition $R$. Let $T^{\epsilon}$ be the first time that the solution $y_{\frac{t}{\epsilon}}$ starting from $y_{0}$ exits $U_{0}$. Set

$$
H_{i}^{\epsilon}(t)=H_{i}\left(y_{t / \epsilon}^{\epsilon}\right) .
$$

(1) Let $\bar{H}(t) \equiv \bar{H}_{t} \equiv\left(\bar{H}_{1}(t), \ldots \bar{H}_{n}(t)\right)$ be the solution to the following system of deterministic equations.

$$
\frac{d}{d t} \bar{H}_{i}(t)=\int_{M_{\bar{H}(t)}} \omega\left(X_{H_{i}}, K\right)(\bar{H}(t), z) d \mu_{\bar{H}_{t}}(z),
$$

with initial condition $\bar{H}(0)=H\left(y_{0}\right)$. Let $T^{0}$ be the first time that $\bar{H}(t)$ exits from $U_{0}$. Then for all $t<T_{0}, \beta>1$, there exists a constant $C_{2}>0$ such that

$$
\left(\mathbf{E}\left(\sup _{s \leq t}\left\|H^{\epsilon}\left(s \wedge T^{\epsilon}\right)-\bar{H}\left(s \wedge T^{\epsilon}\right)\right\|^{\beta}\right)\right)^{\frac{1}{\beta}} \leq C_{2} \epsilon^{1 / 4},
$$

(2) Let $r>0$ be such that $U \equiv\left\{x:\left\|H(x)-H\left(y_{0}\right)\right\| \leq r\right\} \subset U_{0}$ and define

$$
T_{\delta}=\inf _{t}\left\{\left|\bar{H}_{t}-H\left(y_{0}\right)\right| \geq r-\delta\right\} .
$$

Then for any $\beta>1, \delta>0$ and a constant $C$ depending on $T_{\delta}$,

$$
P\left(T^{\epsilon}<T_{\delta}\right) \leq C\left(T_{\delta}\right) \delta^{-\beta} \epsilon^{\beta / 4} .
$$

Remark 3.4. To see that (3.7) is a genuine system of ordinary differential equations, take the canonical transformation map $x_{a}: M_{a} \rightarrow T^{n}$. The pushed forward measure $x_{*}\left(\mu_{a}\right)$ is the Lebesque measure $\mu$ on the torus and (3.7) becomes:

$$
\frac{d}{d t} \bar{H}_{i}(t)=\int_{T^{n}} \omega\left(X_{H_{i}}, K\right)\left(x_{\bar{H}_{t}}^{-1}\left(\bar{H}_{t}, z\right)\right) d \mu(z) .
$$

Proof. By Itô's formula, for $t<T_{0} \wedge T^{\epsilon}$.

$$
H_{i}^{\epsilon}(t)=H_{i}\left(y_{0}\right)+\int_{0}^{t} \omega\left(X_{H_{i}}, K\right)\left(y_{\frac{s}{\epsilon}}^{\epsilon}\right) d s .
$$

For $i$ fixed, write

$$
g_{i}=\omega\left(X_{H_{i}}, K\right)
$$

We only need to estimate

$$
\left|H_{i}^{\epsilon}(t)-\bar{H}_{i}(t)\right|=\left|\int_{0}^{t} g_{i}\left(y_{s / \epsilon}^{\epsilon}\right) d s-\bar{H}_{i}(t)\right|
$$

Using the notation of the previous lemma then equation (3.7) can be written as

$$
\begin{aligned}
\frac{d}{d t} \bar{H}_{i}(t) & =Q^{g_{i}}\left(\bar{H}_{t}\right) \\
\bar{H}_{0} & =H\left(y_{0}\right) .
\end{aligned}
$$

Apply (3.3) to the functions $g_{i}$ we have for any $t<T^{\epsilon}$,

$$
\begin{aligned}
\left|H_{i}^{\epsilon}\left(t \wedge T^{\epsilon}\right)-\bar{H}_{i}\left(t \wedge T^{\epsilon}\right)\right| & \leq \int_{0}^{t \wedge T^{\epsilon}}\left|Q^{g_{i}}\left(H^{\epsilon}(s)\right)-Q^{g_{i}}(\bar{H}(s))\right| d s+\delta\left(g_{i}, \epsilon, t\right) \\
& \left.\leq C(g, \phi) \int_{0}^{t} \| H^{\epsilon}(s)\right)-\bar{H}(s) \| d s+\delta\left(g_{i}, \epsilon, t\right) .
\end{aligned}
$$


By Gronwall's inequality,

$$
\left(\mathbf{E}\left(\sup _{s \leq t \wedge T^{\epsilon}}\left\|H^{\epsilon}(s)-\bar{H}(s)\right\|^{\beta}\right)\right)^{\frac{1}{\beta}} \leq C(t) \epsilon^{\frac{1}{4}},
$$

concluding part (1) of Theorem 3.3

Part (2) of the theorem easily follows. By definition $T_{\delta}$ is the first time that

$$
\sqrt{\sum_{i}\left|\bar{H}_{i}(s)-H_{i}\left(y_{0}\right)\right|^{2}} \geq r-\delta
$$

then

$$
\begin{aligned}
P\left(T^{\epsilon}<T_{\delta}\right) & \leq P\left(\sup _{s \leq T_{\delta} \wedge T^{\epsilon}}\left\|\bar{H}_{s}-H^{\epsilon}(s)\right\|>\delta\right) \\
& \leq \delta^{-\beta} \mathbf{E}\left(\sup _{s \leq T_{\delta} \wedge T^{\epsilon}}\left\|\bar{H}_{i}(s)-H_{i}^{\epsilon}(s)\right\|^{\beta}\right) \\
& \leq C \delta^{-\beta} \epsilon^{\frac{\beta}{4}} .
\end{aligned}
$$

\section{Perturbation by a Hamiltonian Vector Field}

If the perturbation $K$ to the stochastic Hamiltonian system (1.1) is a Hamiltonian vector field, i.e. $L_{X} \omega=0$, then $\int_{M_{c}} \omega\left(X_{H_{i}}, K\right) d \mu_{c}$ vanishes if $c$ is not a bifurcation value. In fact let $\left(U_{0}, \phi\right)$ be an action angle coordinate around $M_{c}$. We can write $K=X_{k}$ for some smooth function $k$,

$$
\begin{aligned}
\int_{M_{c}} \omega\left(X_{H_{i}}, K\right)(z) d \mu_{c}(z) & =\int_{T^{n}} d(k \circ \phi)\left(-\sum_{k=1}^{n} \frac{\partial\left(H_{k} \circ \phi\right)}{\partial I^{k}} \frac{\partial}{\partial \theta_{k}}\right) d \theta \\
& =-\sum_{\beta=1}^{n} \omega_{\beta}^{i}(I) \int_{T^{n}}\left(\frac{\partial}{\partial \theta_{\beta}}\right)(k \circ \phi) d \theta=0,
\end{aligned}
$$

where $d \theta$ is the standard measure on the n-torus. The ordinary differential equation (3.7) governing $\lim _{\epsilon \rightarrow 0} H_{i}\left(y_{t / \epsilon}^{\epsilon}\right)$ has thus a constant solution. In this case we may consider the second order scaling $y_{t / \epsilon^{2}}^{\epsilon}$ and the accumulation of the perturbation over a large time interval of order $\epsilon^{-2}$. The proof is inspired by a proof in Hairer-Pavliotis [15] and this also benefited from the articles by Khasminski, Papanicolau-Stroock-Varadhan and Freidlin.

Let

$$
\mathcal{L}_{0}(I)=\frac{1}{2} \sum \mathbf{L}_{X_{H_{i}}} \mathbf{L}_{X_{H_{i}}}+\mathbf{L}_{V}
$$

be the restriction of the elliptic operator on the invariant manifold with value $I$. If $f$ on $M_{I}$ has $\int f d \mu=0$ then the Poisson equation

$$
\mathcal{L}_{0} h=f
$$

is solvable. We denote by $\mathcal{L}^{-1} f$ the solution to the Poisson equation satisfying $\int \mathcal{L}^{-1} f d \mu=$ 0 .

Since $\mathcal{L}_{0}$ is elliptic on each level set manifold $M_{a}$ and $\left\{H_{i}, k\right\}$ is centered there, the Poisson equation has a unique solution $h_{i}$. Note that the functions $\mathbf{L}_{K}\left\{H_{i}, k\right\}$ and that $\mathbf{L}_{X_{H_{i}}} h_{i}$ are well defined. 
Note that if $K=X_{k}$ then the matrix with $(i, j)$-th entry given by

$$
\left.-d H_{i}(K) \mathcal{L}_{0}^{-1}\left(d H_{j}(K)\right)\right)
$$

is positive definite.

Theorem 4.1. Assume condition $R$ and suppose that $K$ is a smooth local Hamiltonian vector field so that $K=X_{k}$ for some smooth function $k$ in the chart $U_{0}$. Define the matrices $\left(a_{i j}\right)$ by

$$
a_{i j}(a)=-\int_{M_{a}} \omega\left(K, X_{H_{j}}\right) \mathcal{L}_{0}^{-1}\left(\omega\left(K, X_{H_{i}}\right)\right)(a, z) d \mu_{a}(z), \quad a \in D \subset \mathbf{R}^{n}
$$

and let $\left(\sigma_{i}^{j}\right)$ be its square root. Set

$$
b_{j}(a)=\frac{1}{2} \int_{M_{a}} \mathbf{L}_{K} \mathcal{L}_{0}^{-1}\left(\omega\left(X_{H_{j}}, K\right)\right)(a, z) d \mu_{a}(z) .
$$

Let $z_{t}$ be the solution to the following stochastic differential equation

$$
d z_{t}^{j}=\sum_{i} \sigma_{i}^{j}\left(z_{t}\right) \circ d B_{t}^{i}+b_{j}\left(z_{t}\right) d t .
$$

Then the law of the stochastic process $H\left(y_{\frac{t}{\epsilon^{2}}}^{\epsilon}\right)$ stopped at $S^{\epsilon}$, the first time that the process $y_{\frac{t}{\epsilon^{2}}}^{\epsilon}$ exits from $U_{0}$, converges to that of $H\left(z_{t \wedge S^{\epsilon}}\right)$.

Remark: The limiting measure is clearly well defined as $a_{i j}$ and $b_{j}$ are invariant with different choices of the inverse to $\mathcal{L}_{0}$.

Proof. In the following calculation we restrict ourselves on the event $\left\{t<S^{\epsilon}\right\}$, equivalently consider the relevant processes stopped at $S^{\epsilon}$. Set

$$
\begin{aligned}
\hat{y}_{t}^{\epsilon} & =y_{\frac{t}{\epsilon^{2}}}^{\epsilon} S^{\epsilon} \\
\hat{H}^{\epsilon}(t) & \equiv\left(\hat{H}_{1}^{\epsilon}(t), \ldots, \hat{H}_{n}^{\epsilon}(t)\right)=\left(H_{1}\left(\hat{y}_{t}^{\epsilon}\right), \ldots, H_{n}\left(\hat{y}_{t}^{\epsilon}\right)\right) .
\end{aligned}
$$

Then

$$
\hat{H}_{i}^{\epsilon}(t)=H_{i}\left(y_{0}\right)-\epsilon \int_{0}^{\frac{t}{\epsilon^{2}} \wedge S^{\epsilon}} \omega\left(K, X_{H_{i}}\right)\left(y_{s}^{\epsilon}\right) d s .
$$

We first show that the family of the laws $\mu^{\epsilon}$, distribution of $\hat{H}^{\epsilon}\left(t \wedge S^{\epsilon}\right)$, is tight. It follows, by Prohorov's theorem, that $\left\{\mu^{\epsilon}\right\}$ is relatively compact in the space of probability measures with the topology of weak convergence and hence has a weak limit $\bar{\mu}$. To see the tightness of the family $\mu^{\epsilon}$, we show that for any $a, \eta>0$ there is a $\delta>0$ with

$$
P\left(\sup _{|s-t|<\delta}\left\|\hat{H}^{\epsilon}(t)-\hat{H}^{\epsilon}(s)\right\|^{2} \geq a\right) \leq \eta .
$$

For this, observe that

$$
\left\|\hat{H}^{\epsilon}(t)-\hat{H}^{\epsilon}(s)\right\|^{2}=\sum_{i=1}^{n}\left|-\epsilon \int_{\frac{s}{\epsilon^{2}} \wedge S^{\epsilon}}^{\frac{t}{\epsilon^{2}} \wedge S^{\epsilon}} \omega\left(K, X_{H_{i}}\right)\left(y_{r}^{\epsilon}\right) d r\right|^{2} .
$$

Set $h_{i}$ to be the solution to the Poisson equation:

$$
\mathcal{L}_{0} h_{i}=\omega\left(K, X_{H_{i}}\right)
$$


with $\int_{M_{a}} h_{0}=0$ for any $a \in \mathbf{R}^{n}$. Then

$$
\begin{aligned}
& \left\|\hat{H}^{\epsilon}(t)-\hat{H}^{\epsilon}(s)\right\|^{2} \\
= & \sum_{i=1}^{n}\left|\epsilon \sum_{j=1}^{n} \int_{\frac{s}{\epsilon^{2}} \wedge S^{\epsilon}}^{\frac{t}{\epsilon^{2}} \wedge S^{\epsilon}} \mathbf{L}_{X_{H_{j}}} h_{i}\left(y_{r}^{\epsilon}\right) d B_{r}^{j}+\epsilon^{2} \int_{\frac{s}{\epsilon^{2}} \wedge S^{\epsilon}}^{\frac{t}{\epsilon^{2}} \wedge S^{\epsilon}} \mathbf{L}_{K} h_{i}\left(y_{r}^{\epsilon}\right) d r-\epsilon h_{i}\left(\hat{y}_{t}^{\epsilon}\right)+\epsilon h_{i}\left(\hat{y}_{s}^{\epsilon}\right)\right|^{2} .
\end{aligned}
$$

Applying Lemma 3.2 with $\epsilon$ replaced by $\epsilon^{2}$, one see that the drift term has a nice bound in $|t-s|:$

$$
\begin{aligned}
& \epsilon^{2} \int_{\frac{s}{\epsilon^{2}} \wedge S^{\epsilon}}^{\frac{t}{\epsilon^{2}} \wedge S^{\epsilon}} \mathbf{L}_{K} h_{i}\left(y_{r}^{\epsilon}\right) d r \\
& =\int_{s \wedge \tau^{\epsilon}}^{t \wedge \tau^{\epsilon}} \int_{\hat{H}^{\epsilon}(r)} \mathbf{L}_{K} h_{i}(z) d \mu(z) d r+\delta\left(\mathbf{L}_{K} h_{i}, \epsilon, t-s\right),
\end{aligned}
$$

This gives us a comfortable estimates since $\delta\left(\mathbf{L}_{K} h_{i}, \epsilon, t-s\right)$ is of the order $\sqrt{\epsilon}(t-s)$. Similarly the quadratic variation of each of martingale terms also converges with the same rate of convergence:

$$
\mathbf{E}\left\langle\int_{\frac{s}{\epsilon^{2}} \wedge S^{\epsilon}}^{\frac{t}{\epsilon^{2}} \wedge S^{\epsilon}} \mathbf{L}_{X_{H_{j}}} h_{i}\left(y_{r}^{\epsilon}\right) d B_{r}^{j}\right\rangle=\epsilon^{2} \int_{s \wedge \tau^{\epsilon}}^{t \wedge \epsilon} \int \mathbf{E}\left|\mathbf{L}_{X_{H_{j}}} h_{i}\left(y_{r}^{\epsilon}\right)\right|^{2} d r .
$$

Applying Burkerholder-Gundy inequality to obtain an estimate on the $L_{\beta}$ norm of

$$
\sup _{|s-t|<\delta} \epsilon\left|\sum_{j=1}^{n} \int_{\frac{s}{\epsilon^{2}} \wedge S^{\epsilon}}^{\frac{t}{\epsilon^{2}} \wedge S^{\epsilon}} \mathbf{L}_{X_{H_{j}}} h_{i}\left(y_{r}^{\epsilon}\right) d B_{r}^{j}\right|,
$$

which is a constant multiple of $|s-t|$ plus an error term of the order $\sqrt{\epsilon}(t-s)$.

Finally it is clear that

$$
\sup _{|s-t|<\delta}\left|\epsilon h_{i}\left(\hat{y}_{t}^{\epsilon}\right)-\epsilon h_{i}\left(\hat{y}_{s}^{\epsilon}\right)\right|^{2} \leq C \epsilon \rightarrow 0 .
$$

To identify the limiting measure let $h$ be the solution to the Poisson equation,

$$
h=\frac{1}{2} \sum_{i=1}^{n} \partial_{i} F(H) \mathcal{L}_{0}^{-1}\left(\omega\left(K, X_{H_{i}}\right)\right),
$$

where $\mathcal{L}_{0}^{-1}$ is considered to act on the angle variable only and $\int_{M_{a}} h=0$ for each $a$. For any smooth function $F$ on $\mathbf{R}^{n}$, we have

$$
\begin{aligned}
& \left.F\left(\hat{H}^{\epsilon}(t)\right)\right)-F\left(\hat{H}^{\epsilon}(0)\right) \\
& =-\epsilon \sum_{i=1}^{n} \int_{0}^{\frac{t}{\epsilon^{2}} \wedge S^{\epsilon}} \partial_{i} F\left(H\left(y_{s}^{\epsilon}\right)\right) \omega\left(K, X_{H_{i}}\right)\left(y_{s}^{\epsilon}\right) d s \\
& =\epsilon \sum_{j=1}^{n} \int_{0}^{\frac{t}{\epsilon^{2}} \wedge S^{\epsilon}} \mathbf{L}_{X_{H_{j}}} h\left(y_{s}^{\epsilon}\right) d B_{s}^{j}+\epsilon^{2} \int_{0}^{\frac{t}{\epsilon^{2}} \wedge S^{\epsilon}} \mathbf{L}_{K} h\left(y_{s}^{\epsilon}\right) d s+\epsilon\left(h\left(y_{0}\right)-h\left(\hat{y}_{t}^{\epsilon}\right)\right) .
\end{aligned}
$$

The first term on the right hand side is a martingale and the last term converges to zero as $\epsilon \rightarrow 0$. We first identify $\mathbf{L}_{K} h$ in terms of the function $F$. By assumption the functions 
$\omega\left(K, X_{H_{i}}\right)$ are centred and $\mathcal{L}_{0}^{-1}$ has no effect on functions of $H$ and so

$$
\begin{aligned}
\mathbf{L}_{K} h= & \frac{1}{2} \mathbf{L}_{K} \mathcal{L}_{0}^{-1}\left(\sum_{i=1}^{n} \partial_{i} F(H) \omega\left(K, X_{H_{i}}\right)\right) \\
= & \frac{1}{2} \mathbf{L}_{K}\left(\sum_{i=1}^{n}\left(\partial_{i} F\right)(H) \mathcal{L}_{0}^{-1}\left(\omega\left(K, X_{H_{i}}\right)\right)\right) \\
= & -\frac{1}{2} \sum_{i=1}^{n} \partial_{j} \partial_{i} F(H) \omega\left(K, X_{H_{j}}\right) \mathcal{L}_{0}^{-1}\left(\omega\left(K, X_{H_{i}}\right)\right) \\
& +\frac{1}{2}\left(\sum_{i=1}^{n} \partial_{i} F(H) \mathbf{L}_{K} \mathcal{L}_{0}^{-1}\left(\omega\left(X_{H_{i}}, K\right)\right)\right)
\end{aligned}
$$

Set

$$
\overline{\mathcal{L}}=-\frac{1}{2} \sum_{i, j} \omega\left(K, X_{H_{j}}\right) \mathcal{L}_{0}^{-1}\left(\omega\left(K, X_{H_{i}}\right)\right) \partial_{i} \partial_{j}+\frac{1}{2} \sum_{i=1}^{n} \mathbf{L}_{K} \mathcal{L}_{0}^{-1}\left(\omega\left(K, X_{H_{i}}\right)\right) \partial_{i}
$$

to see

$$
\begin{aligned}
& \left.F\left(\hat{H}^{\epsilon}(t)\right)\right)-F\left(\hat{H}^{\epsilon}(0)\right) \\
= & \epsilon \sum_{j=1}^{n} \int_{0}^{\frac{t}{\epsilon^{2}} \wedge S^{\epsilon}} \mathbf{L}_{X_{H_{j}}} h\left(y_{s}^{\epsilon}\right) d B_{s}^{j}+\epsilon^{2} \int_{0}^{\frac{t}{\epsilon^{2}} \wedge S^{\epsilon}} \overline{\mathcal{L}} F \circ H\left(y_{s}^{\epsilon}\right) d s+\epsilon\left(h\left(y_{0}\right)-h\left(\hat{y}_{t}^{\epsilon}\right)\right) .
\end{aligned}
$$

Mimicking Papanicolaou-Stroock-Varadhan, we define $\mathcal{F}_{s}^{H} \equiv \sigma\left\{\hat{H}_{r \wedge S^{\epsilon}}^{\epsilon}: r \leq s\right\}$ and so $\left\{\mathcal{F}_{s}^{H}: s \geq 0\right\}$ is the filtration generated by $\hat{H}_{\cdot \wedge S^{\epsilon}}^{\epsilon}$. We need the following estimates:

$$
\begin{aligned}
& \epsilon^{2} \int_{a \wedge T^{\epsilon}}^{\frac{t}{\epsilon^{2} \wedge S^{\epsilon}}} \overline{\mathcal{L}} F\left(H\left(y_{s}^{\epsilon}\right)\right) d s \\
= & \int_{a \wedge T^{\epsilon}}^{t \wedge T^{\epsilon}}\left(\int_{M_{\hat{H}^{\epsilon}(s)}} \overline{\mathcal{L}} F \circ H(z) d \mu_{\hat{H}^{\epsilon}(s)}(z)\right) d s+\delta\left(\overline{\mathcal{L}} F, \epsilon^{2}, t-a\right) \\
= & \int_{a \wedge T^{\epsilon}}^{t \wedge T^{\epsilon}} \mathcal{L} F \circ \hat{H}^{\epsilon}(s) d s+\delta\left(\overline{\mathcal{L}} F \circ H, \epsilon^{2}, t-a\right),
\end{aligned}
$$

where in the action-angle local coordinate,

$$
\begin{aligned}
\mathcal{L} F(a)= & -\frac{1}{2} \sum_{i, j=1}^{n} \partial_{j} \partial_{i} F(a) \int_{M_{a}} \omega\left(K, X_{H_{j}}\right) \mathcal{L}_{0}^{-1}\left(\omega\left(K, X_{H_{i}}\right)\right)(a, z) d \mu_{I}(z) \\
& +\frac{1}{2} \sum_{i=1}^{n} \partial_{i} F(a) \int_{M_{a}} \mathbf{L}_{K} \mathcal{L}_{0}^{-1}\left(\omega\left(X_{H_{i}}\right), K\right)(a, z) d \mu(z)
\end{aligned}
$$


Then for any $\mathcal{F}_{s}^{H}$ - measurable $L^{2}$ random function $G$, using again Lemma 3.2 ,

$$
\begin{aligned}
& \left.\left.\mathbf{E} G\left[F\left(\hat{H}^{\epsilon}\left(t \wedge S^{\epsilon}\right)\right)\right)-F\left(\hat{H}^{\epsilon}\left(s \wedge S^{\epsilon}\right)\right)\right)-\int_{s \wedge S^{\epsilon}}^{t \wedge S^{\epsilon}} \int_{M_{\hat{H}^{\epsilon}(r)}}(\overline{\mathcal{L}} F)(z) d \mu_{\hat{H}^{\epsilon}(r)}(z) d r\right] \\
& =\mathbf{E} G\left[\epsilon \sum_{j=1}^{n} \int_{\frac{s}{\epsilon^{2}} \wedge S^{\epsilon}}^{\frac{t}{\epsilon^{2}} \wedge S^{\epsilon}} \mathbf{L}_{X_{H_{j}}} h\left(y_{s}^{\epsilon}\right) d B_{s}^{j}\right] \\
& +\mathbf{E} G\left[\delta\left(\overline{\mathcal{L}} F, \epsilon^{2}, t-s\right)+\epsilon\left(h\left(y_{\frac{s}{\epsilon^{2}} \wedge S^{\epsilon}}^{\epsilon}\right)-h\left(y_{\frac{t}{\epsilon^{2}} \wedge S^{\epsilon}}^{\epsilon}\right)\right)\right] \\
& =\mathbf{E} G\left[\delta\left(\overline{\mathcal{L}} F, \epsilon^{2}, t-s\right)+\epsilon\left(h\left(y_{\frac{s}{\epsilon^{2}}}^{\epsilon} \wedge S^{\epsilon}\right)-h\left(y_{\frac{t}{\epsilon^{2}}}^{\epsilon} \wedge S^{\epsilon}\right)\right)\right] \rightarrow 0 .
\end{aligned}
$$

Consequently

$\left.\left.\mathbf{E}\left\{F\left(\hat{H}^{\epsilon}\left(t \wedge S^{\epsilon}\right)\right)\right)-F\left(\hat{H}^{\epsilon}\left(s \wedge S^{\epsilon}\right)\right)\right)-\int_{s \wedge S^{\epsilon}}^{t \wedge S^{\epsilon}} \int_{M_{\hat{H}^{\epsilon}(r)}}(\overline{\mathcal{L}} F)(z) d \mu_{\hat{H}^{\epsilon}(r)}(z) d r \mid \mathcal{F}_{s}^{H}\right\} \rightarrow 0$,

and so any weak limit of the law $\hat{H}^{\epsilon}$. is the solution to the martingale problem for the second order differential operator $\mathcal{L}$.

Acknowledgement. This research has benefitted from inspiring discussions with Martin Hairer. I would also like to thank R. Hudson and the referees for critical reading and comments.

\section{REFERENCES}

[1] L. Arnold, P. Imkeller, and N. Sri Namachchivaya. The asymptotic stability of a noisy non-linear oscillator. J. Sound Vibration, 269(3-5):1003-1029, 2004.

[2] V. I. Arnold, V. V. Kozlov, and A. I. Neřshtadt. Mathematical aspects of classical and celestial mechanics. In Dynamical systems, III, volume 3 of Encyclopaedia Math. Sci. Springer, Berlin, 1993.

[3] R. Abraham and J.E. Marsden. Foundations of Mechanics, second edition. The Benjamin/Cummings publishing Company, 1978.

[4] V. I. Arnold. Mathematical methods of classical mechanics, volume 60 of Graduate Texts in Mathematics. Springer-Verlag, second edition, 1989. Translated from the Russian by K. Vogtmann and A. Weinstein.

[5] J.-M. Bismut. Mécanique aléatoire, volume 866 of Lecture Notes in Mathematics. Springer-Verlag, Berlin, 1981. With an English summary.

[6] A. Borodin and M. Freidlin, M. Fast oscillating random perturbations of dynamical systems with conservation laws. Ann. Inst. H. Poincaré Probab. Statist. 31(3): 485-525, 1995.

[7] D, Dolgopyat. Evolution of additive invariants in stochastic averaging. Stoch. Dyn. 4(2):265-275, (2004).

[8] A. Eizenberg and M. I. Freidlin. Averaging principle for perturbed random evolution equations and corresponding Dirichlet problems. Probab. Theory Related Fields, 94(3):335-374, 1993.

[9] K. D. Elworthy, Y. Le Jan, and Xue-Mei Li. On the geometry of diffusion operators and stochastic flows, volume 1720 of Lecture Notes in Mathematics. Springer-Verlag, Berlin, 1999.

[10] M. I. Freidlin and A. D. Wentzell. Diffusion processes on graphs and the averaging principle. Ann. Probab., 21(4):2215-2245, 1993.

[11] M. I. Freidlin and A. D. Wentzell. Random perturbations of dynamical systems, volume 260 of Grundlehren der Mathematischen Wissenschaften [Fundamental Principles of Mathematical Sciences]. Springer-Verlag, New York, second edition, 1998. Translated from the 1979 Russian original by Joseph Szücs.

[12] M. Freidlin and M. Weber On random perturbations of Hamiltonian systems with many degrees of freedom. Stochastic Process. Appl. 94 (2001), no. 2, 199-239.

[13] M. Freidlin and M. Weber Random perturbations of dynamical systems and diffusion processes with conservation laws. Probab. Theory Related Fields 128 (3) : 441-466, 2004.

[14] D. Givon, R. Kupferman, and A. Stuart. Extracting macroscopic dynamics: model problems and algorithms. Nonlinearity, 17(6):R55-R127, 2004. 
[15] M. Hairer and G. A. Pavliotis. Periodic homogenization for hypoelliptic diffusions. J. Statist. Phys., 117(12):261-279, 2004.

[16] H. Hofer and E. Zehnder. Symplectic invariants and Hamiltonian dynamics. Birkhäuser Advanced Texts: Basler Lehrbücher. [Birkhäuser Advanced Texts: Basel Textbooks]. Birkhäuser Verlag, Basel, 1994.

[17] R. Z. Khas'minskii. The behavior of a conservative system under the action of slight friction and slight random noise. J. Appl. Math. Mech., 28:1126-1130 (1965), 1964.

[18] R. Khasminskii and N. Krylov. On averaging principle for diffusion processes with null-recurrent fast component. Stochastic Process. Appl., 93(2):229-240, 2001.

[19] L. Koralov. Random perturbations of 2-dimensional Hamiltonian flows. Probab. Theory Related Fields, 129(1):37-62, 2004.

[20] R. Z. Khasminskii and G. Yin. On averaging principles: an asymptotic expansion approach. SIAM J. Math. Anal., 35(6):1534-1560 (electronic), 2004

[21] D. McDuff and D. Salamon. Introduction to symplectic topology. Oxford Mathematical Monographs. The Clarendon Press Oxford University Press, New York, 1995. Oxford Science Publications.

[22] G. C. Papanicolaou. Asymptotic analysis of stochastic equations. In Studies in probability theory, volume 18 of MAA Stud. Math., pages 111-179. Math. Assoc. America, Washington, D.C., 1978.

[23] G. C. Papanicolaou, D. Stroock, and S. R. S. Varadhan. Martingale approach to some limit theorems. In Papers from the Duke Turbulence Conference (Duke Univ., Durham, N.C., 1976), Paper No. 6, pages ii+120 pp. Duke Univ. Math. Ser., Vol. III. Duke Univ., Durham, N.C., 1977.

[24] R. B. Sowers. Stochastic averaging with a flattened Hamiltonian: a Markov process on a stratified space (a whiskered sphere). Trans. Amer. Math. Soc., 354(3):853-900 (electronic), 2002.

[25] N. Srinamachchivaya and R. B. Sowers. Rigorous stochastic averaging at a center with additive noise. Meccanica, 37:85-114, 2002.

Department of Mathematics, University of Warwick, Coventry CV4 7AL, U.K. 